\title{
Local Features of the Fermi Surface Curvature and the Anomalous Skin Effect in Metals
}

\author{
Natalya A. Zimbovskaya \\ Department of Physics and Electronics, University of Puerto Rico at Humacao, Humacao, PR 00791
}

(Dated: November 7, 2018)

\begin{abstract}
In this paper we present a theoretical analysis of the effect of local geometrical structure of the Fermi surface on the surface impedance of a metal at the anomalous skin effect. We show that when the Fermi surface includes nearly cylindrical and/or flattened segments it may significantly change both magnitude and frequency dependence of the surface impedance. Being observed in experiments these unusual frequency dependencies could bring additional information concerning fine geometrical features of the Fermi surfaces of metals.

PACS numbers: $73.21 \mathrm{Cd} ; 73.40 .-\mathrm{C}$
\end{abstract}

\section{INTRODUCTION}

It is well known that electromagnetic waves incident at the surface of a metal cannot penetrate deeply inside. Actually, the field inside the metal vanishes at the distances of the order of $\delta$ from the surface. This effect is called the skin effect, and the characteristic depth $\delta$ is called the skin depth. The suppression of the electromagnetic field inside the metal originates from the response of conduction electrons, and it occurs when the frequency $\omega$ of the incident wave is smaller than the electrons plasma frequency $\omega_{p}$. The latter is the characteristic frequency for the response of the conduction electrons system to an external disturbance. When $\omega>\omega_{p}$ the electrons are too slow to respond, and the electromagnetic field penetrates into the metal without decay. Due to the skin effect the incident electromagnetic field could affect condition electrons only when they move inside the layer of the thickness $\delta$ near the metal surface. The skin depth depends on the electric conductivity of the metal $\sigma$ and on the frequency $\omega$ of the incident wave as well. Increase in $\sigma$ and/or $\omega$ leads to the decrease in the skin depth. At high frequencies $\tau^{-1} \ll \omega \ll \omega_{p}(\tau$ is the scattering time for conduction electrons) and low temperatures, $\delta$ may become smaller than the electrons mean free path $l$. When the condition $\delta<l$ is satisfied the effect is referred to as the anomalous skin effect. At the anomalous skin effect the response of a metal to an incident electromagnetic wave is determined with the electrons moving in the skin layer nearly in parallel with the surface of the metal sample. These "efficient" electrons are associated with a few small "effective segments" on the Fermi surface (FS). The remaining electrons stay in the skin layer only for a very short while which prevents them from responding to the electromagnetic field.

A theory of the anomalous skin effect in metals was first proposed more than five decades ago by A. B. Pippard [1] and G. E. Reuter and E. H. Zondheimer [2], and R. B. Dingle 3] using an isotropic model for a metal. The main results of these studies were presented in some books where high frequency phenomena in metals were discussed [4]. Then the theory was further developed to make it applicable to realistic metals with anisotropic Fermi surfaces [5, 6, 7, 8, 9]. It became clear that the response of conduction electrons to an external electromagnetic field under the anomalous skin effect depends on the Fermi surface (FS) geometry, especially its Gaussian curvature $K(\mathbf{p})=1 / R_{1}(\mathbf{p}) R_{2}(\mathbf{p})$, where $R_{1,2}(\mathbf{p})$ are the principal radii of curvature. For the most of real metals FSs are complex in shape, and their curvature turns zero at some points. These points could be partitioned in two classes. First, these exist zero curvature points where only one of the principal radii has a singularity, whereas another one remains finite. Usually, such points are combined in lines of zero curvature. The latter are either inflection lines or they label positions of nearly cylindrical strips on the FSs. Also, some points could be found where both principal radii tend to infinity. These points are set out separately, and the FSs are flattened in their vicinities.

When a FS includes points of zero curvature it leads to an enhancement of the contribution from the neighborhoods of these points to the electron density of states (DOS) on the FS. Normally, this enhanced contribution is small compared to the main term of the DOS which originates from the major part of the FS. Therefore it cannot produce noticeable changes in the response of the metal when all segments of the FS contribute essentially equally. However, when the curvature turns zero at some points on an "effective" part of the FS, it can give a sensible enhancement in the number of efficient electrons and, in consequence, a pronounced change in the response of the metal to the disturbance.

It has been shown that when the FS includes nearly cylindrical and/or flattened segments, noticeable changes may be observed in the frequency and temperature dependencies of sound dispersion and absorption 10, 11, 12, 13. Also, the shape and amplitude of quantum oscillations in various characteristics of a metal could be affected by of the FS local geometry in the vicinities of the extremal cross sections. Qualitative anomalies in the de Haas-van Alphen oscillations associated with cylindri- 
cal pieces of the FSs were considered in Refs. [14, 15]. Similar anomalies in quantum oscillations in the static elastic constants and the velocity of sound were analyzed in [16, 17.

Here, we concentrate on the analysis of possible manifestations of the FS local geometry in the surface impedance of a metal at the anomalous skin effect. In this case the main contribution to the surface impedance of a metal originates from electrons moving nearly in parallel with the surface of the metal. These electrons are efficient quasiparticles, and they belong to the "effective" part of the FS. The effects of the FS geometry on the metal response at the anomalous skin effect were analyzed before [9, 18] adopting some simplified models for the FS. The purpose of the present work is to carry out a general analysis whose results are independent on particularities in energy-momentum relations and could be applied to a broad class of metals.

\section{RESULTS AND DISCUSSION}

We consider a metal filling the half-space $z<0$. A plane electromagnetic wave is incident on the metal surface making a right angle with the latter. To analyze the response of the metal to the wave we calculate the surface impedance:

$$
Z_{\alpha \beta}=E_{\alpha}(0) / \int_{0}^{-\infty} J_{\beta}(z) d z
$$

Here, $\alpha, \beta=x, y ; E_{\alpha}(z)$ and $J_{\beta}(z)$ are the components of the electric field $\mathbf{E}$ and electric current density $\mathbf{J}$, respectively. Considering the anomalous skin effect we can limit our analysis to the case of specular reflection of electrons from the surface. Then the surface impedance tensor has the form:

$$
Z_{\alpha \beta}=\frac{8 i \omega}{c^{2}} \int_{0}^{\infty}\left(\frac{4 \pi i \omega}{c^{2}} \sigma-q^{2} I\right)_{\alpha \beta}^{-1} d q
$$

Here, $\omega$ and $\mathbf{q}$ are the frequency and the wave vector of the incident wave, respectively $(\mathbf{q}=(0,0, q)) ; \sigma$ is the electron conductivity tensor, and $I_{\alpha \beta}=\delta_{\alpha \beta}$.

To proceed we assume that the FS has a mirror symmetry in a momentum space relative to a plane $p_{z}=0$. To simplify calculations of the electron conductivity we divide each sheet of the FS in segments in such a way that the momentum $\mathbf{p}$ is a one-to-one function of the electron velocity $\mathbf{v}$ over a segment. The segments may coincide with the FS sheets. Also, it could happen that some sheets include a few segments. This depends on the FS shape. In calculation of the conductivity we carry out integration over each segment using spherical coordinates in the velocity space, namely, the velocity magnitude at the $j$-th segment $v_{j}$, and the spherical angles $\theta, \varphi$. So, the element of the surface area is given by the expression: $d A_{j}=\sin \theta d \theta d \varphi /\left|K_{j}(\theta, \varphi)\right|$ where $K_{j}(\theta, \varphi)$ is the Gaussian curvature of the $j$-th FS segment. Summing up contributions from all these segments we obtain:

$$
\begin{aligned}
& \sigma_{\alpha \beta}(\omega, q)=\frac{i e^{2}}{4 \pi^{3} \hbar^{3} q} \sum_{j} \int d \varphi \\
& \times \int \frac{n_{\alpha} n_{\beta} \sin \theta d \theta}{\left|K_{j}(\theta, \varphi)\right|\left[(\omega+i / \tau) / q v_{j}-\cos \theta\right]} .
\end{aligned}
$$

Here, $n_{\alpha, \beta}=v_{j \alpha, \beta} / v_{j}$, and $\tau$ is the electron scattering time. The limits in the integrals over $\theta, \varphi$ are determined with the shape of the segments. We remark, however, that the effective strips on the FS are determined by the condition $v_{z} \approx 0$ for efficient electrons move in parallel with the metal surface at $z=0$. Therefore, the upper limit in the integral over $\theta$ in the terms corresponding to the segments including the effective strips must equal $\pi / 2$. In the following calculations we omit the term $i / \tau$ in the Eq. (3) assuming $\omega \tau \gg 1$ which is typical for the anomalous skin effect in good metals. Using Eq. (3) we can easily write out the expressions for the conductivity components. We have:

$$
\begin{aligned}
& \sigma_{x x}(\omega, q)=\frac{i e^{2}}{4 \pi^{3} \hbar^{3} q} \sum_{j} \int d \varphi \\
& \times \int \frac{d \theta \cos ^{2} \varphi \sin ^{3} \theta}{\left|K_{j}(\theta, \varphi)\right|\left[(\omega+i / \tau) / q v_{j}-\cos \theta\right]} .
\end{aligned}
$$

Another conductivity component $\sigma_{y y}$ is described with the similar expression where $\cos ^{2} \varphi$ in the integrand numerator is replaced by $\sin ^{2} \varphi$. In further calculations we assume for simplicity that the chosen $z$ axis coincides with a high symmetry axis for the FS, so that both conductivity and surface impedance tensors are diagonalized.

The main contribution to the surface impedance under the anomalous skin effect comes from the region of large $q$ where $\omega / q v \ll 1$. To calculate the corresponding asymptotic expressions for the conductivity components we expand the integrand in the Eq. (4) in powers of $\omega / q v$. Then we can write the well known result for the principal term in the expansion of the conductivity component $\sigma_{x x}(\omega, q)$ :

$$
\sigma_{0}(q)=\frac{e^{2}}{4 \pi^{3} \hbar^{3} q} \sum_{l} \int d \varphi \frac{\cos ^{2} \varphi}{\left|K_{l}(\pi / 2, \varphi)\right|} \equiv \frac{e^{2}}{4 \pi \hbar^{3} q} p_{0}^{2}
$$

The same asymptotics could be obtained for $\sigma_{y y}$, so the indices are omitted for simplicity here and in following expressions. Summation over $l$ is carried out over all segments of the FS containing effective strips which correspond to $\theta=\pi / 2\left(v_{z}=0\right)$ and the curvature $K_{l}(\pi / 2, \varphi)$ is supposed to take finite and nonzero value at any point of any effective strip. For a spherical FS $p_{0}$ equals the 
Fermi momentum $p_{F}$. In realistic metals the two are not equal but have the same order of magnitude. In general, $p_{0}$ is determined by the Eq. (5). Then we can calculate the next term in the expansion of conductivity in powers of $\omega / q v$. For a FS whose curvature everywhere is finite and nonzero we arrive at the result

$$
\sigma_{1}(\omega, q)=\sigma_{0}(q) \frac{i \omega}{q v_{0}} .
$$

Here, the velocity $v_{0}$ has the order of the Fermi velocity $v_{F}$ :

$$
\begin{aligned}
\frac{1}{v_{0}} & =\frac{2}{\pi^{2} p_{0}^{2}} \sum_{l} \int d \varphi \int_{\alpha_{l}}^{\pi / 2} \frac{d \theta \cos ^{2} \varphi \sin \varphi}{\cos ^{2} \theta} \\
& \times\left[\frac{1+\cos ^{2} \theta / \cos ^{2} \alpha_{l}}{\left|K_{l}(\pi / 2, \varphi)\right| v_{l}(\pi / 2, \varphi)}-\frac{\sin ^{2} \theta}{\left|K_{l}(\theta, \varphi)\right| v_{l}(\theta, \varphi)}\right] \\
& -\frac{2}{\pi^{2} p_{0}^{2}} \sum_{j \neq l} \int d \varphi \int_{\theta<\pi / 2} \frac{d \theta \cos ^{2} \varphi \sin ^{3} \theta}{\left|K_{j}(\theta, \varphi)\right| v_{j}(\theta, \varphi) \cos ^{2} \theta} .
\end{aligned}
$$

Here, the lower limit $\alpha_{l}$ in the integral over $\theta$ in the first term takes on values determined by the FS shape $\left(\alpha_{l}<\right.$ $\pi / 2)$. The second term corresponds to the contribution from the FS segments which do not include effective lines. For the spherical FS we have $v_{0}=\pi v_{F} / 4$.

When the curvature at any effective line turns zero, it changes the asymptotics for the conductivity, as we show below. First, we assume that the curvature becomes zero at a whole effective line passing through one of the segments of the Fermi surface. Keeping in mind that $z$ axis in the chosen reference system runs along a high order symmetry axis we can present the relevant energymomentum relation in the form:

$$
E(\mathbf{p})=E_{1}\left(p_{x}, p_{y}\right)+E_{2}\left(p_{z}\right) .
$$

Near the effective line where $v_{z} \equiv \partial E_{2} / \partial p_{z}=0$ we can approximate $E_{2}\left(p_{z}\right)$ as follows:

$$
E_{2}\left(p_{z}\right) \approx E_{0}\left(\frac{p_{z}-p^{*}}{p^{*}}\right)^{2 l} ; \quad l \geq 1 .
$$

Here, $E_{0}, p^{*}$ have the dimensions of the energy and momentum, respectively; $p_{z}=p^{*}$ corresponds to the effective line.

In the vicinity of the effective line we can write the following expression for the curvature of the FS corresponding to the Eq. (8):

$$
K(\mathbf{p})=\frac{1}{v^{4}} \frac{\partial v_{z}}{\partial p_{z}}\left(v_{y}^{2} \frac{\partial v_{x}}{\partial p_{x}}+v_{x}^{2} \frac{\partial v_{y}}{\partial p_{y}}-2 v_{x} v_{y} \frac{\partial v_{x}}{\partial p_{y}}\right) .
$$

The value of the curvature at $v_{z}=0$ is determined by the factor $\partial v_{z} / \partial p_{z} \sim\left[\left(p_{z}-p^{*}\right) / p^{*}\right]^{2 l-2}$. The curvature becomes zero at the effective line when $l>1$. Expressing this factor as a function of velocity (which is necessary to carry out integration over a region in the velocity space) we get $\partial v_{z} / \partial p_{z} \sim\left(v_{z}\right)^{-\beta}$ where $\beta=-1+1 /(2 l-1)$.

So, we can use the following approximation for the curvature $K(\theta, \varphi)$ at $\theta \leq \pi / 2$ :

$$
K(\theta, \varphi)=W(\theta, \varphi)(\cos \theta)^{-\beta},
$$

In this expression, the function $W(\theta, \varphi)$ everywhere assumes finite and nonzero values, and the exponent $\beta$ takes on negative values which correspond to the line of zero curvature at $\theta=\pi / 2$. In the close vicinity of this line the FS is nearly cylindrical in shape. The closer $\beta$ to -1 , the closer to a cylinder is the effective strip on the FS. The contribution to the conductivity from the nearly cylindrical segment on the FS is given by:

$$
\begin{aligned}
& \sigma_{a}(\omega, q)=\frac{i e^{2} \omega}{2 \pi^{3} \hbar^{3} q}\left[\int d \varphi \int _ { \alpha } ^ { \pi / 2 } d \theta \left(\frac{\sin ^{2} \theta}{|W(\theta, \varphi)| v(\theta, \varphi)}\right.\right. \\
& \left.-\frac{1}{|W(\pi / 2, \varphi)| v(\theta, \varphi)}\right) \frac{\cos ^{2} \varphi \sin \theta(\cos \theta)^{\beta}}{(\omega / q v(\theta, \varphi))^{2}-\cos ^{2} \theta} \\
& \left.+\int \frac{d \varphi \cos ^{2} \varphi}{|W(\pi / 2, \varphi)| v(\pi / 2, \varphi)} \int_{\alpha}^{\pi / 2} \frac{d \theta \sin \theta(\cos \theta)^{\beta}}{(\omega / q v(\theta, \varphi))^{2}-\cos ^{2} \theta}\right] .
\end{aligned}
$$

Using this asymptotic expression we can calculate the "anomalous" contribution to the conductivity $\sigma_{a}(\omega, q)$ for small $\omega / q v$. Introducing the largest magnitude of the velocity on the effective line $v_{a}$ we have:

$$
\begin{aligned}
\sigma_{a}(\omega, q) & =\rho \sigma_{0}(q)\left(\frac{\omega}{q v_{a}}\right)^{\beta}\left[1-i \tan \left(\frac{\pi \beta}{2}\right)\right], \\
\rho & \approx \frac{1}{\pi p_{0}^{2}} \int \frac{d \varphi \cos ^{2} \varphi}{|W(\pi / 2, \varphi)|}
\end{aligned}
$$

Comparing Eq. (14) with the definition for $p_{0}^{2}$ introduced earlier by Eq. (5) we see that $\rho$ is a dimensionless factor whose value is determined with the relative number of the "effective" electrons concentrated at the nearly cylindrical effective segment.

The value of the contribution to the conductivity from the "anomalous" effective strip depends on the character of the curvature anomaly at given strip, and on the relative number of effective electrons concentrated here as shown in the Fig. 1. In this figure we display plots of $\left|\sigma(\omega, q) / \sigma_{0}(q)\right| \equiv\left|1+\sigma_{a}(\omega, q) / \sigma_{0}(q)\right|$ versus $\omega / q v$. When the parameter $\rho$ takes on values of the order or greater than 0.1 (the number of effective electrons associated with the anomalous sections on the FS is comparable to the total number of the effective electrons), the term $\sigma_{a}(\omega, q)$ can predominate over $\sigma_{0}(q)$ and determine the conductivity value at large $q$. This occurs when the shape parameter $\beta$ accepts values not too close to 

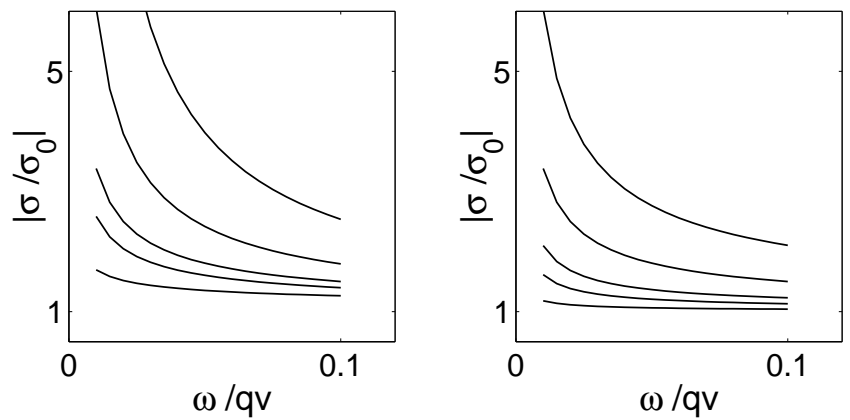

FIG. 1: Conductivity component $\sigma_{x x}(\omega, q)$ including the contribution from a zero-curvature segment on the FS at large $q(\omega / q v \ll 1)$. Left panel: the curves are plotted at $\rho=0.2$ and $\beta=-0.8,-0.7,-0.6,-0.4,-0.2$ from the top to the bottom. Right panel: the curves are plotted at $\beta=-0.6$ and $\rho=0.2,0.1,0.05,0.03,0.01$ from the top to the bottom.

zero, and the curvature anomaly at the effective line is well pronounced. When either $\rho$ or $\beta$ or both are very small in magnitude, the main approximation to the conductivity is described with Eq. 5 as well as for a metal whose FS curvature is everywhere nonzero. Nevertheless, in such cases the term $\sigma_{a}(\omega, q)$ also is important for it gives the first correction to the principal term in the expression for the conductivity.

Also, the anomalous contribution to the conductivity could appear when the FS is flattened at some points belonging to an effective segment. To avoid lengthy calculations we illustrate the effect of such points on the conductivity using a simple expression representing the energy momentum relation near the point of flattening $\mathcal{M}_{0}\left(p_{1}, 0,0\right)$ :

$$
E(\mathbf{p})=\frac{p_{1}^{2}}{2 m_{1}}\left(\frac{p_{x}^{2}}{p_{1}^{2}}\right)+\frac{p_{2}^{2}}{m_{2}}\left(\frac{p_{y}^{2}+p_{z}^{2}}{p_{2}^{2}}\right)^{l}
$$

where $p_{1}, p_{2}$ have dimensions of momentum. When $l=1$ this expression corresponds to the ellipsoidal FS, and $m_{1}, m_{2}$ are the principal values of the effective mass tensor. The FS curvature equals:

$$
\begin{aligned}
& K(\mathbf{p})=\frac{l}{m_{2} v^{4}}\left(\frac{p_{y}^{2}+p_{z}^{2}}{p_{2}^{2}}\right)^{l-1} \\
& \times\left[\frac{1}{m_{1}}\left(v_{y}^{2}+v_{z}^{2}\right)+v_{x}^{2} \frac{l(2 l-1)}{m_{2}}\left(\frac{p_{y}^{2}+p_{z}^{2}}{p_{2}^{2}}\right)^{l-1}\right] .
\end{aligned}
$$

For $l>1$ the curvatures of both principal cross sections of the FS become zero at the point $\left(p_{1}, 0,0\right)$ indicating the FS local flattening.

Turning to the spherical coordinates in the velocity space we can rewrite the expression (16) in the form:

$$
K(\theta, \varphi)=W(\theta, \varphi)\left(\cos ^{2} \theta+\sin ^{2} \varphi\right)^{(1-\beta) / 2}
$$

where the shape parameter $\beta=-1+2 /(2 l-1)$. When $l>1$, the FS curvature becomes zero at $\theta=\pi / 2, \varphi=0$ which correspond to the point $\mathcal{M}_{0}$. The parameter $\beta$ takes on values from the interval $(-1,1)$, and the more pronounced is the FS flattening near the point $\mathcal{M}$ ( the greater is the value of $l$ ) the closer is $\beta$ to -1 . The "anomalous" contribution to the conductivity originating from the flattened segment of the FS has the form similar to Eq. (13), namely:

$$
\sigma_{a}(\omega, q)=\mu \sigma_{0}(q)\left(\frac{\omega}{q v(\pi / 2,0)}\right)^{\beta}\left[1-i \tan \left(\frac{\pi \beta}{2}\right)\right] .
$$

Here, $\mu$ is a small dimensionless factor proportional to the relative number of conduction electrons associated with the flattened part of the FS. Due to the smallness of $\mu$ the term (17) may be significant only when $\beta \leq$ $0(l \geq 1.5)$. Otherwise, it could be neglected.

Now, we proceed in calculations of the surface impedance given by the expression (2). Under anomalous skin effect conditions the impedance can be represented as an expansion in inverse powers of the anomaly parameter $(\xi \gg 1)$. Representing the conductivity as the sum of terms (5) and (6), we can calculate two first terms in the expansion of the surface impedance in inverse powers of the anomaly parameter:

$$
\begin{aligned}
Z & \equiv R-i H=-\frac{8 i \omega}{c^{2}} \delta \int_{0}^{\infty} d t \frac{1}{1-i t^{3}(1+i t / \xi)} \\
& \approx Z_{0}\left(\frac{\omega}{\omega_{0}}\right)^{2 / 3}\left[1-i \sqrt{3}-\frac{2}{3}\left(\frac{\omega}{\omega_{0}}\right)^{2 / 3}(1+\sqrt{3})\right]
\end{aligned}
$$

where $\delta=\left(c^{2} \hbar^{3} / e^{2} p_{0}^{2} \omega\right)^{1 / 3}$ is the skin depth,

$$
Z_{0}=\frac{8 \pi}{3 \sqrt{3}} \frac{v_{0}}{c^{2}} ; \quad \xi=\frac{v_{0}}{\omega \delta} \equiv\left(\frac{\omega}{\omega_{0}}\right)^{2 / 3}
$$

is the anomaly parameter, and frequency $\omega_{0}$ equals:

$$
\omega_{0}=\left(\frac{v_{0}}{\hbar}\right)^{3 / 2} \frac{e p_{0}}{c} .
$$

Keeping in mind that $v_{0} \sim v_{F}$ and $p_{0} \sim p_{F}$ we can roughly estimate the characteristic frequency $\omega_{0}$. In good metals the electron density has the order of $10^{21}-10^{22} \mathrm{~cm}^{-3}$, so $\omega_{0} \sim 10^{12}-10^{13} \mathrm{~s}^{-1}$. This is significantly smaller that the plasma frequency $\omega_{p}$ which in good metals is of the order of $10^{15}-10^{16} s^{-1}$. As one would expect, the inequality $\omega \ll \omega_{0}(\xi \gg 1)$ agrees with the general requirement on frequencies $\omega \ll \omega_{p}$, and could be satisfied at $\omega \sim 10^{10}-10^{11} \mathrm{~s}^{-1}$.

The expression of the form (19) was first obtained by R. B. Dingle (see [3]) within the isotropic model of metal. Later it was generalized to be applied to realistic metals, assuming that their FSs do not include segments of zero 

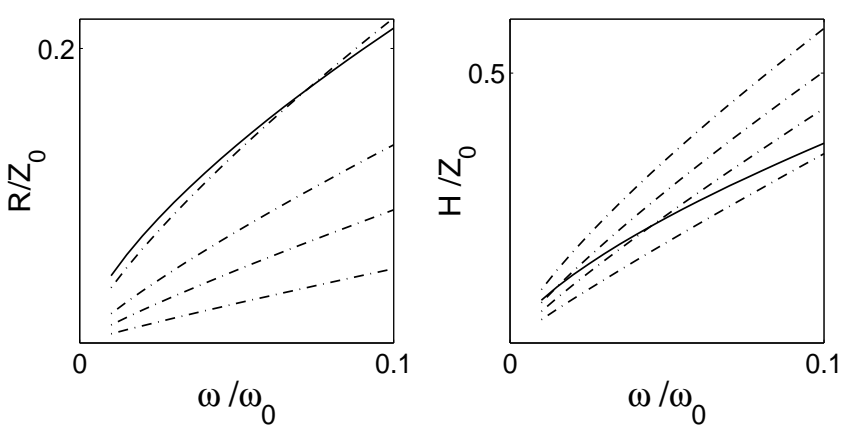

FIG. 2: Frequency dependence of the real $(R)$ and imaginary $(H)$ parts of the surface impedance of a metal under the anomalous skin effect. Dashed-dotted lines are plotted using Eq.(12) at $\rho=0.2$ and $\beta=-0.8,-0.7,-0.6,-0.4$, from the top to the bottom. Solid lines represent real and imaginary parts of the surface impedance of a metal whose FS does not include nearly cylindrical and/or flattened segments.

curvature [8]. For such FSs the frequency dependence of the surface impedance has the same character, as for a Fermi sphere. The main approximation of the surface impedance is proportional to $\omega^{2 / 3}$ and the first correction to it is proportional to $\omega^{4 / 3}$.

When the FS includes locally flattened or nearly cylindrical segments the asymptotic expression for the surface impedance changes. The effect of this anomalous local geometry of the FS on the impedance is especially strong for $\beta<-0.5, \rho \gtrsim 0.1$. Under these conditions the "anomalous" contribution dominates over the other terms in the expression for conductivity and determines the principal term of the surface impedance. As a result we have:

$$
Z \approx Z_{0} \zeta(\beta)\left(\frac{\omega}{\omega_{0}}\right)^{2 /(3+\beta)}
$$

where

$$
\begin{aligned}
\zeta(\beta) & =\frac{3 \sqrt{3} \rho^{-1 / 3+\beta}}{3+\beta}\left(\cos \frac{\pi \beta}{2}\right)^{1 /(3+\beta)} \\
& \times\left[\cot \left(\frac{\pi}{3+\beta}\right)-i\right] .
\end{aligned}
$$

The surface impedance described with (21) differs in magnitude from that of a conventional metal whose FS does not include zero curvature segments. Frequency dependence of the surface impedance also changes as shown in the Fig. 2. Now it is proportional to $\omega^{2 /(\beta+3)}$. For a nearly cylindrical effective strip the exponent $2 /(\beta+3)$ varies in the interval $(0.8,1)$ where the value 1 corresponds to the precisely cylindrical strip. So, when the significant part of effective electrons is associated with a nearly cylindrical effective strip, the impedance should
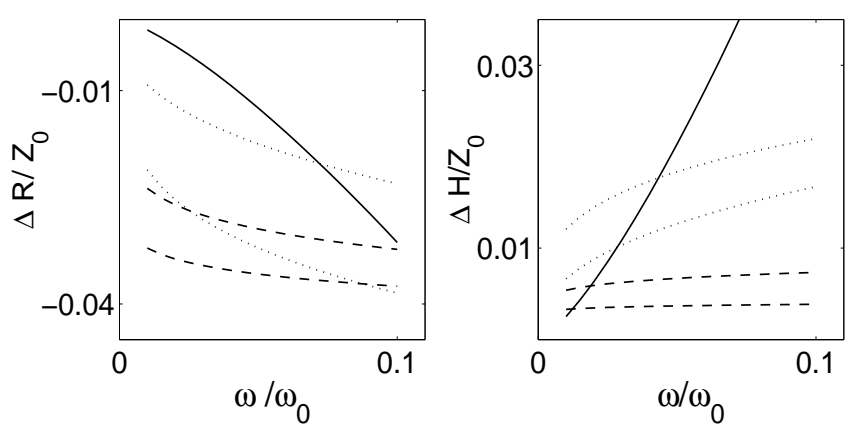

FIG. 3: Frequency dependence of the real $(\Delta R)$ and imaginary $(\Delta H)$ parts of the first correction to the main term in the surface impedance expansion in the inverse powers of the anomaly parameter. The curves are plotted at $\rho=0.01, \beta=-0.9 ; \rho=0.02, \beta=-0.8$ (dashed lines); $\rho=0.1, \beta=-0.5 ; \rho=0.1, \beta=-0.4$ (dotted lines). Solid lines present the real and imaginary parts of the first correction to the main approximation for the impedance of a metal whose FS does not include nearly cylindrical and/or flattened segments.

slower increase with increase of frequency than in a "conventional" case.

Now we consider more realistic case when either $\rho$ or $\beta$ or both take on values close to zero (a zero curvature segment on the effective part of the FS is narrow and/or the curvature anomaly is only moderately pronounced). In this case the anomalous contribution (13) is the first correction to the main approximation for the conductivity, and it determines the first correction to the approximation for the surface impedance:

$$
Z=Z_{0}\left(\frac{\omega}{\omega_{0}}\right)^{2 / 3}(1-i \sqrt{3})+\Delta Z
$$

Here,

$$
\begin{aligned}
\Delta Z & \equiv \Delta R-i \Delta H \approx-Z_{0} \eta(\beta)\left(\frac{\omega}{\omega_{0}}\right)^{2(\beta+1) / 3} ; \quad \\
\eta(\beta) & =\frac{\rho(\beta+1)}{\sqrt{3}} \frac{1}{\cos (\pi \beta / 2)}\left\{\cot \left(\frac{\pi(\beta+1)}{3}\right)+i\right\} .
\end{aligned}
$$

We can apply this result (24) to describe the contribution to the surface impedance from narrow or weakly developed nearly cylindrical strip and also from a point of flattening located on the effective segment of the FS. The correction to the main approximation of the surface impedance now is proportional to $\omega^{2(\beta+1) / 3}$ as we show in the Fig. 3. The presented analysis shows that the surface impedance of a semiinfinite metal whose FS has points or lines of zero curvature can be described by the formulas (21)-(24). The obtained asymptotic expressions indicate that an anomaly of curvature on an effective line 
changes frequency dependence of the surface impedance, and under certain conditions it can essentially change its magnitude. This follows from the discussed above relation between the curvature of the FS and the number of effective electrons.

\section{CONCLUSION}

The concept of Fermi surface is recognized as one of the most meaningful concepts in condensed matter physics, providing an excellent insight in the main electronic properties of conventional metals and other materials with metallic-like conductivity. Extensive studies based on experimental data concerning effects responsive to the structure of electronic spectra in metals and using advanced computational methods were carried out to restore the FS geometries. These efforts were resulted in the impressive mapping of the FSs of conventional metals. However, in the course of these studies a comparatively little attention was paid to fine local features in the FS geometries including zero curvature lines and/or points of flattening. These local curvature anomalies do not significantly affect main geometrical characteristics of FSs (such as connectivity, locations of open orbits, sizes and arrangements of sheets) which are usually determined from the standard experiments. Therefore these local features could be easily missed when a FS is restored, if one does not expect them to be present, and does not pay special attention to keep them in the resulting FS image. So, it is important to explore possible experimental manifestations of the FS curvature anomalies. Adoption of the phenomenological models is justified in these studies as far as these models are based on reasonable assumptions concerning the FS geometry. Actually, phenomenological models were commonly used to develop the theory of "standard" effects such as de Haas-van Alphen effect, which were (and are) employed as tools to obtain informations concerning FSs shapes [19]. This approach to fermiology does not contradict that one based on electron band structure calculations. It supplements the latter. Such supplementing analysis could bring new insight in the physical nature and origin of some physical effects including these considered in the present work, and show their usefulness in studies of the FSs geometries.

As for the particular models adopted in the present work they may be reasonably justified within a nearly free electron approximation. Adopting the nearly freeelectrons approach we arrive at the energy-momentum relation for conduction electrons:

$$
E=\frac{\mathbf{k}^{2}}{2 m}+\frac{\mathbf{g}^{2}}{2 m}-\frac{1}{m} \sqrt{(\mathbf{k} \cdot \mathbf{g})^{2}+m^{2} V^{2}},
$$

where $m$ is the effective mass, $\mathbf{k}=\mathbf{g}-\mathbf{p} ; \mathbf{g}=\mathbf{h G} / 2 ; \mathbf{G}$ is a reciprocal lattice wave vector; $V$ is Fourier compo- nent of the potential energy of electron in the lattice field which corresponds to the vector G. Within the nearly free-electron model the energy $V$ is assumed to be small compared to the Fermi energy $E_{F}$, so we introduce a small parameter $\epsilon=\sqrt{V / E_{F}}$.

The corresponding FS looks like a sphere with "knobs" located at those segments which are close to the boundaries of the Brillouin zone. Inflection lines of zero curvature pass along the boundaries between the knobs and the main body of the FS. A FS segment including a knob and its vicinity is axially symmetric, and the symmetry axis is directed along the corresponding reciprocal lattice vector. In further analysis we single out such segment to consider it separately. For certainty we choose the coordinate system whose " $x$ " axis is directed along the reciprocal lattice vector. Within the chosen segment the FS curvature is described with the expression:

$$
K=\frac{m^{2} v_{x}^{2}+p_{\perp}^{2} d v_{x} / d p_{x}}{\left(p_{\perp}^{2}+m^{2} v_{x}^{2}\right)^{2}}
$$

where $p_{\perp}^{2}=p_{y}^{2}+p_{z}^{2}$.

Equating the FS curvature to zero, and using the energy-momentum relation (26) we find the values $p_{x 0}$ and $p_{\perp 0}$ corresponding to the inflection line. We get:

$$
p_{x 0}=p_{F}\left(1-\epsilon^{2} / \sqrt{2}\right), \quad p_{\perp 0} \approx p_{F} \epsilon,
$$

where $p_{F}$ is the radius of the original Fermi sphere. Now, we can expand the variable $p_{x}$ in powers of $\left(p_{\perp}-p_{\perp 0}\right)$ near the zero curvature line. Taking into account that $d^{2} p_{x} / d p_{\perp}^{2}$ turns zero at points belonging to the inflection line, and keeping the lowest-order terms in the expansion, we obtain:

$$
p_{x} \approx p_{x 0}-\frac{\epsilon}{\sqrt{2}}\left(p_{\perp}-p_{\perp 0}\right)-\frac{p_{F}}{\sqrt{2} \epsilon}\left(\frac{p_{\perp}-p_{\perp 0}}{p_{F}}\right)^{3} .
$$

Substituting this approximation into Eq. (26) we arrive at the following energy-momentum relation:

$$
E(\mathbf{p})=\frac{p_{x}^{2}}{2 m}+\frac{2}{\epsilon} \frac{p_{F}^{2}}{2 m}\left(\frac{p_{\perp}-p_{\perp 0}}{p_{F}}\right)^{3} .
$$

The latter could be employed near the zero curvature line where $p_{\perp 0} \ll p_{F}$. Omitting $p_{\perp 0}$ we arrive at the energymomentum relation of the form (15) where $l=3 / 2$. Also, we can compare the equations describing crosssections $p_{y}=0$ of the FS corresponding to the Eq. (30) and our phenomenological model (8), (9). Again, we see that these equations are in agreement with each other provided that $l=3 / 2$.

There is an experimental evidence that "necks" connecting quasispherical pieces of the FS of copper include nearly cylindrical belts [19]. It is also likely that the FS of gold possesses the same geometrical features for it closely resembles that of copper. As for possible flattening of the FS, experiments of [20, 21, 22] on the cyclotron 
resonance in a magnetic field normal to the metal surface give grounds to conjecture that such anomalies could be found on the FSs of cadmium, zinc and even potassium. Another group of materials where we can expect the FS curvature anomalies to be manifested includes layered structures with metallic-type conductivity (e.g. $\alpha-(B E D T-T T F)_{2} M h g(S C H)_{4}$ group of organic metals). Fermi surfaces of these materials are sets of rippled cylinders, isolated or connected by links. There exists experimental evidence that the quasi-two-dimensional FSs of some organic metals include segments with zero curvature 23]. Also, recent investigations give grounds to expect the FSs of some new conducting materials include flattened segments [24, 25, 26].

The most important result of the present work is that it shows how such fine geometric features as points of flattening and/or zero curvature lines could be manifested in experiments on the anomalous skin effect. It is shown that when the FS includes nearly cylindrical segments or it is flattened at some points, qualitative changes may occur in frequency dependencies of the surface impedance under the anomalous skin effect. Being observed in experiments, such unusual frequency dependencies would indicate the presence of zero-curvature lines and points on the FS, and display their location. Also, analyzing these frequency dependencies, the shape parameter $\beta$ could be found giving additional information on the FSs local structure. This information may be used in further studies of the FSs geometries.

\section{ACKNOWLEDGMENTS}

The author thanks G. M. Zimbovsky for help with the manuscript. This work was supported by NSF Advance program SBE-0123654, nsf-prem 0353730 and PR Space Grant NGTS/40091.

[1] A. B. Pippard, Proc. Roy. Soc. A 191, 385 (1947).

[2] G. E. Reuter and E. H. Zondheimer, Proc. Roy. Soc. A 195, 336 (1949).

[3] R. B. Dingle, Physics 19, 311 (1953).
[4] See e.g.: A. B. Pippard, The Dynamic of Conduction Electrons (Gordon and Breach, New York, 1965); P. M. Platzman and P. A. Wolff, Waves and Interactions in Solid State Plasmas (Academic Press, new York, 1973); A. A. Abrikosov, Introduction to the Theory of Normal Metals (Academic Press, New York, 1972); I. M. Lifshitz, M. Ya. Azbel, and M. I. Kaganov, Electron Theory of Metals (Consultants Bureau, New York, 1973); N. A. Zimbovskaya, Local Geometry of the Fermi Surface and High-Frequency Phenomena in Metals (Springer-Verlag, New York, 2001).

[5] A. B. Pippard, Proc. Roy. Soc. A224, 273 (1954).

[6] E. H. Sondheimer, Proc. Roy. Soc. A224, 160 (1954).

[7] M. L. Glasser, Phys. Rev. 176, 1110 (1968).

[8] V. V. Ustinov and D. T. Khusainov, Sov. J. Low. Temp. Phys. 11, 617 (1985).

[9] M. I. Kaganov and P. Kontreras, JETP 79, 360 (1994).

[10] G. T. Avanesjan, M. I. Kaganov and T. Yu. Lisovskaya, JETP, 48, 900 (1978).

[11] V. M. Kontorovich and N. A. Stepanova, JETP 49, 321 (1978).

[12] I. M. Suslov, Sov. Phys. Solid State 23, 1114 (1981).

[13] N. A. Zimbovskaya, JETP 80, 932 (1995).

[14] G. Lacueva and A. W. Overhauser, Phys. Rev. Lett. 46, 1273 (1992).

[15] Ya. M. Blanter, M. I. Kaganov and D. B. Posvyanskii, Phys-Usp. 165, 178 (1995).

[16] N. A. Zimbovskaya, J. Low Temp. Phys. 21, 217 (1995).

[17] N. A. Zimbovskaya, Phys. Rev. B 71, 024109 (2005).

[18] M. I. Kaganov and Yu. V. Gribkova, J. Low Temp. Phys. 17, 473 (1991).

[19] D. Shoenberg, Magnetic Oscillations in Metals (Cambridge University Press, New York, 1984).

[20] V. P. Naberezhnykh and N. K. Dan'shin, JETP 29, 658 (1969).

[21] V. P. Naberezhnykh and V. L. Mel'nik, Sov. Phys. Solid State 7, 197 (1965).

[22] G. A. Baraff, C. C. Grimes and P. M. Platzman, Phys. Rev. Lett. 22, 590 (1969).

[23] J. Wosnitza, Fermi Surface of Low-Dimensional Organic Metals and Superconductors (Springer-Verlag, Berlin, 1996).

[24] C. Bergemann, S. R. Julian, A. P. Mackenzie, S. NishiZaki, and Y. Maeno, Phys. Rev. Lett. 84, 2662 (2000).

[25] Y.-D. Chuang, A. D. Gromko, D. S. Dessau, T. Kimura and Y. Tokura, Science, 292, 1509 (2001).

[26] T. Dahm and N. Schopohli, Phys. Rev. Lett. 91, 017001 (2003). 